\title{
"Las atendemos de lunes a viernes": nacimientos según días de la semana en la Ciudad Autónoma de Buenos Aires, 2004-2013
}

\author{
"We see them from Monday to Friday:" Births by day \\ of the week in the Autonomous City of Buenos Aires, \\ 2004-2013
}

Patricia Rosemberg ${ }^{1}$, Marcio Alazraqui ${ }^{2}$, Hugo Spinelli ${ }^{3}$

\begin{abstract}
${ }^{1}$ Autora de correspondencia. Magíster en Epidemiología, Gestión y Políticas de Salud. Doctoranda en Salud Colectiva. Directora, Carrera de Especialización en Gestión en Salud, Instituto de Salud Colectiva, Universidad Nacional de Lanús, Buenos Aires, Argentina. $\triangle$ (iD)

${ }^{2}$ Doctor en Salud Colectiva. Director, Carrera de Especialización de Epidemiología, Instituto de Salud Colectiva, Universidad Nacional de Lanús, Buenos Aires, Argentina. $\square$ (iD)

${ }^{3}$ Doctor en Salud Colectiva. Director, Instituto de Salud Colectiva, Universidad Nacional de Lanús, Buenos Aires, Argentina. $\triangle$ (iD)
\end{abstract}

RESUMEN El objetivo de este trabajo es describir los nacimientos según su distribución en días de la semana a fin de caracterizar el aspecto programado o no del proceso de parto y nacimiento. Se realizó un estudio epidemiológico descriptivo de la distribución temporal de los días de la semana en que ocurrieron los nacimientos en la Ciudad Autónoma de Buenos Aires, para el período 2004-2013, a partir de la base de datos del Informe Estadístico de Nacido Vivo de la Dirección de Estadísticas e Información de Salud del Ministerio de Salud de la Nación. Se analizaron las siguientes variables: establecimiento (público o privado), edad gestacional, edad y nivel de instrucción de la madre. El análisis muestra que los nacimientos disminuyen significativamente los sábados y domingos en los establecimientos públicos y privados, siendo mayor la disminución en los establecimientos privados; que los nacimientos en la semana 37 son más frecuentes en los establecimientos privados y disminuyen en los días no laborables; y que a mayor nivel de instrucción materna son menores los nacimientos durante los fines de semana. Analizar el día de la semana en el que ocurren los nacimientos transparenta la medicalización del proceso de parto e indicaría que la programación e inducción de los nacimientos ocurre en los establecimientos privados en mayor medida que en los establecimientos públicos.

PALABRAS CLAVES Nacido Vivo; Parto; Periodicidad; Servicios de Salud; Medicalización; Argentina.

\begin{abstract}
The objective of this article is to describe births according to their distribution by day of the week in order to characterize the scheduled or non-scheduled aspect of the labor and delivery process. A descriptive epidemiological study of the temporal distribution of the days of the week on which births occurred in the Autonomous City of Buenos Aires was carried out for the period 2004-2013, based on the Statistical Reports of Live Births database of the Directorate of Statistics and Health Information at the National Ministry of Health. The following variables were analyzed using descriptive statistics: establishment (public or private sector), gestational age, maternal age, and mother's level of education. The analysis reveals that births decrease significantly on Saturdays and Sundays in both public and private establishments, with a greater decrease being observed in private establishments. Births at week 37 are more frequent in private establishments and decrease on non-working days. For mothers with higher levels education, fewer births occurred on weekends. Analyzing the day of the week on which births occur reveals the medicalization of the delivery process and could indicate that the scheduling and induction of births occur in private establishments to a greater extent than in public establishments.
\end{abstract}

KEY WORDS Live Birth; Parturition; Periodicity; Health Services; Medicalization; Argentina. 


\section{INTRODUCCIÓN}

Este artículo se propone describir la medicalización del parto y del nacimiento tomando como indicador los días de la semana en que ocurren y el tipo de institución en que se realizan.

Es esperable que los nacimientos ocurran en proporciones similares en cada uno de los siete días de la semana, siguiendo una distribución de probabilidad uniforme discreta. En este sentido, cuando se cumple ese tipo de distribución, la proporción esperada de nacimientos para cada día de la semana es del $14,28 \%{ }^{(1)}$. Por lo tanto, el día de la semana en el que se produce el nacimiento, podría verse modificado únicamente por su programación. Existe poca evidencia científica de la distribución por días de la semana de nacimientos y los cambios que ha experimentado en el tiempo. En función de este problema nos preguntamos ¿cómo es la distribución en días de la semana de los nacimientos en establecimientos públicos y privados de la Ciudad Autónoma de Buenos Aires (CABA) entre los años 2004 y 2013?

La hipótesis es que la distribución semanal de los nacimientos en CABA no es uniforme, sino que es mayor de lunes a viernes y disminuye los sábados y domingos; y que estas diferencias en la distribución son más marcadas en los establecimientos privados. La programación de los nacimientos se debe a intereses económicos y agendas laborales de las y los profesionales, que son priorizadas por sobre el cuidado de la mujer y de la niña o el niño recién nacido, quienes quedan subordinados a la medicalización del parto y el nacimiento. Describir los nacimientos según la distribución en los días de la semana y dónde se producen permite caracterizar un aspecto de la medicalización del proceso de parto y nacimiento.

Inducir o programar el parto antes de término nos permite reflexionar acerca de la información y el diálogo de las y los profesionales con las mujeres en la elección del tipo de parto y nacimiento de sus hijas e hijos $^{(2)}$. La gestión de los recursos sanitarios, los riesgos inherentes al embarazo ${ }^{(3)}$, la fatiga de la madre $y$, especialmente, la adaptación al entorno laboral han favorecido -junto con otros aspectos de carácter social- un control más activo por parte de las y los profesionales sanitarios del momento del nacimiento(4).

La autoridad médica se refleja en la programación de los nacimientos, que pasó de ser un proceso humanizado y fisiológico a un acto institucionalizado, gobernado por médicas y médicos, quienes utilizan su hegemonía acomodando el proceso a sus intereses y agendas individuales o corporativas, con la internalización y naturalización por parte de las mujeres de la medicalización del parto ${ }^{(5)}$.

La exagerada intervención médica, el uso abusivo de la tecnología y la medicalización del embarazo pueden condicionar el proceso de parto y nacimiento, y determinar algunos aspectos de la salud de la mujer y el niño ${ }^{(6,7)}$. La cascada de intervenciones médicas encuentra consenso en el concepto de riesgo asociado al embarazo y, a partir de allí, las prácticas médicas se mecanizan durante el proceso perinatal y en la atención del parto, y se pasa a considerar que todos los embarazos implican algún riesgo(2).

Las sociedades occidentales atraviesan cambios sociales que provocan importantes transformaciones culturales, que determinan prácticas y comportamientos de las médicas y los médicos que asisten a partos programados y cesáreas o, incluso, de mujeres que tras quedar embarazadas asumen que quieren programar una cesárea y no pasar por un parto ${ }^{(8,9)}$.

Las diferencias en las prácticas del parto y nacimiento se pueden explicar más por factores socioculturales que biológicos ${ }^{(1)}$. Así, la programación de los partos y nacimientos mediante inducciones del trabajo de parto o cesáreas se ve influenciada también por factores no médicos como presión social, intereses económicos o rutinas de trabajo, que se justifican con el pretexto de indicaciones clínicas ${ }^{(10)}$.

A pesar que desde diferentes organismos oficiales y sociedades científicas se ha trabajado por un enfoque del parto menos medicalizado $^{(11)}$, nos encontramos ante un nuevo fenómeno social que se ha dado en Ilamar el "nacimiento a la carta"(12). 
Este artículo fue presentado como parte de la calificación del proyecto de tesis del Doctorado en Salud Colectiva de la Universidad Nacional de Lanús, Argentina, siendo la doctoranda la magíster Patricia Rosemberg, su director el doctor Hugo Spinelli y su codirector el doctor Marcio Alazraqui.

\section{METODOLOGÍA}

Se realizó un estudio epidemiológico descriptivo de la tendencia temporal de los días de la semana en que ocurren los nacimientos en CABA, para el período 2004-2013.

Se utilizó la base de datos del Informe Estadístico de Nacido Vivo, del Subsistema de Estadísticas Vitales, provista por la Dirección de Estadísticas e Información de Salud del Ministerio de Salud de la Nación. Como se puede observar en el instructivo del instrumento de recolección ${ }^{(13)}$, esta base de datos no releva la vía de finalización del parto (vaginal o cesárea), por lo que las variables seleccionadas fueron: establecimiento, edad gestacional, edad de la madre, nivel de instrucción materna y fecha del nacimiento.

Los criterios de selección fueron todos los nacimientos ocurridos en establecimientos públicos o privados en CABA entre los años 2004 y 2013, con edad gestacional entre 34 y 41 semanas. Se denominaron nacimientos a los registros de nacidos vivos de la base de datos del Informe Estadístico de Nacido Vivo.

La elección de CABA se debió a la calidad de los datos disponibles, puesto que al realizar la fase exploratoria de los datos incluidos en el Informe Estadístico de Nacido Vivo, se comprobó que el campo fecha de nacimiento contaba con el día de ocurrencia en un $97,5 \%$ de los registros en CABA, en contraposición al resto del país, donde los valores eran menores.

Respecto al tipo de establecimiento, se analizaron los nacimientos ocurridos en establecimientos públicos y privados y se excluyeron los ocurridos en vivienda $(0,2 \%)$ y otros lugares como, por ejemplo, la vía pública $(0,1 \%)$.
En cuanto a la edad gestacional se agruparon de acuerdo con lo descripto por la Organización Mundial de la Salud (OMS), que considera prematuro a un recién nacido vivo antes de que se haya cumplido 37 semanas de gestación ${ }^{(14)}$. En la base de datos del Informe Estadístico de Nacido Vivo, la edad gestacional se informa -la mayoría de las veces- de acuerdo con la fecha de la última menstruación y cuando no se encuentra disponible se utiliza su determinación por ecografía precoz o por examen físico al nacer.

En el análisis de edad gestacional, se incluyeron los recién nacidos con edad gestacional de 34 a 36 semanas, considerados prematuros tardíos, y los recién nacidos con edad gestacional de 37 a 41 semanas, considerados de término(14). Se excluyeron del análisis los menores de 34 semanas, por considerar menos probable la inducción o programación del parto por causas no médicas.

Se define a los recién nacidos con edad gestacional entre las 37 y 38 semanas de gestación como término precoz (early term) (RNTP) y a los recién nacidos con edad gestacional de 39 a 41 semanas como término completo (full term) (RNTC) ${ }^{(15)}$.

Para analizar la instrucción materna, según el máximo nivel de instrucción alcanzado, se agruparon en: a) secundario incompleto o menor (que incluye las mujeres que nunca asistieron a la escuela, las que realizaron el primario de forma incompleta, las que finalizaron el primario y las que asistieron al secundario pero no lo finalizaron); y b) secundario completo o superior (que incluye las mujeres que finalizaron el secundario, las que alcanzaron la instrucción superior o universitaria pero no la completaron, y las que completaron el nivel superior o universitario). En la fase exploratoria se observó una diferencia relevante a partir de este agrupamiento de los niveles de instrucción.

Se desestimaron los niveles con denominación educación general básica (EGB 1, 2 y 3) completo o incompleto y polimodal (completo o incompleto) debido a que esos niveles de instrucción no se implementaron en CABA y correspondían a menos del $1 \%$ de los datos (5.905 mujeres). 
Para analizar cada una de las variables descriptas (tipo de establecimiento, edad gestacional, instrucción y edad materna) se realizó un análisis bivariado tomando como variable agrupada el día de la semana del nacimiento.

Se analizó la relación entre el nivel de instrucción de la madre y el día de ocurrencia del nacimiento en establecimientos públicos y privados por separado, a través del test chi cuadrado $\left(\chi^{2}\right)$ de Pearson, y se calculó el valor de $p$. Para el análisis de los datos se utilizó el programa Microsoft Excel 2013 ${ }^{\odot}$.

Las bases de datos se confeccionaron y analizaron manteniendo las regulaciones éticas del secreto estadístico contemplados en la Ley 17622/68, que garantiza la confidencialidad y preservación de la identidad de las personas involucradas o entidades a quienes se refiera. Se respetó la no divulgación y/o utilización de información que pueda dar lugar a la identificación de personas particulares ${ }^{(16)}$.

\section{RESULTADOS}

Se registraron 822.891 nacimientos institucionales entre los años 2004 y 2013 en CABA (Tabla 1), de los cuales el 41,1\% (338.396 nacimientos) ocurrieron en establecimientos públicos y el 58,9\% (484.495 nacimientos) en establecimientos privados.

$\mathrm{Al}$ analizar las edades maternas, observamos que, en el grupo entre 21 y 35 años, ocurrió el $71,3 \%$ de los nacimientos totales; en el grupo de 31 a 35 años, el $26,7 \%$; y en el de 26 a 30 años, el 25,4\%. Con relación al nivel de instrucción materna, observamos que el grupo de mujeres con secundario completo o superior alcanzó el $61,4 \%$ de los nacimientos descriptos. En cuanto a la edad gestacional, el $75,2 \%$ de los niños nacieron en las semanas 38,39 y 40 ; el $19,4 \%$, en la semana 38 ; el $26,3 \%$, en la semana $39 ;$ y un $29,5 \%$, en la semana 40. La distribución de los nacimientos en establecimientos públicos y privados fue diferente según la edad materna. Así, en el grupo de mujeres de 25 años o menores, observamos que el $68,0 \%$ de los nacimientos totales ocurrió en los establecimientos públicos, mientras que en el grupo de mujeres mayores de 25 años, los nacimientos en establecimientos privados alcanzaron el 71,4\%.

En cuanto al nivel de instrucción materna se observa que, en los establecimientos públicos, el $66,0 \%$ de las mujeres tenía un nivel de instrucción bajo (secundario incompleto o menor); en cambio, en los establecimientos privados, el $83,7 \%$ de las mujeres alcanzó un nivel de instrucción alto.

El 58,9\% de los nacimientos ocurrió en establecimientos privados, y al analizar la distribución porcentual público-privada observamos que se modifica según la edad gestacional. Del total de los nacimientos pretérminos tardíos (semanas 34,35 y 36 ) el $65,7 \%$ ocurrió en establecimientos privados; de los nacimientos de término precoz (semanas 37 y 38) el 69,7\%, en establecimientos privados; mientras que, de los nacimientos de término completo (semanas 39, 40 y 41 ), el $54,0 \%$ ocurrió en establecimientos privados.

Para analizar la distribución por tipo de establecimiento y días de la semana, según edad gestacional, se utilizó el test de significación chi cuadrado, y se observa que, tanto en establecimientos públicos como privados, no sigue una distribución uniforme discreta, que se correspondería a un valor del 14,28\% para cada día de la semana ${ }^{(1)}$.

En la Tabla 2, observamos la distribución por días de la semana y edad gestacional tanto en establecimientos públicos como privados, y las mayores diferencias porcentuales se presentan en edades gestacionales tempranas, en las que los nacimientos considerados pretérmino -que por riesgo poblacional (en cuanto a edad y nivel de instrucción materna) se esperaría que ocurran en los establecimientos públicos- tienen una mayor ocurrencia en los privados y muestran mayor diferencia para la distribución de lunes a viernes que el mismo grupo de nacimientos en los establecimientos públicos.

Para los nacimientos llamados de término precoz (early term) -recién nacidos de 37 y 38 semanas-, en la Tabla 2 se observa que las niñas y los niños que nacen en la semana 37 , en establecimientos privados, el 
Tabla 1. Número de nacimientos institucionales y distribución porcentual según sexo, edad materna, nivel de instrucción materna y edad gestacional en establecimientos públicos y privados. Ciudad Autónoma de Buenos Aires, 2004-2013.

\begin{tabular}{|c|c|c|c|c|c|c|}
\hline \multirow[t]{2}{*}{ Variable } & \multicolumn{2}{|c|}{ Total nacimientos } & \multicolumn{2}{|c|}{ Establecimientos públicos } & \multicolumn{2}{|c|}{ Establecimientos privados } \\
\hline & $\mathrm{n}$ & $\%$ & $\mathrm{n}$ & $\%$ & $n$ & $\%$ \\
\hline \multicolumn{7}{|l|}{ Sexo } \\
\hline Masculino & 421.787 & 51,3 & 173.227 & 51,2 & 248.560 & 51,3 \\
\hline Femenino & 401.090 & 48,7 & 165.159 & 48,8 & 235.931 & 48,7 \\
\hline Indeterminado & 14 & 0,0 & 10 & 0,0 & 4 & 0,0 \\
\hline \multicolumn{7}{|l|}{ Edad materna (en años) } \\
\hline 15 o menos & 4.712 & 0,6 & 3.872 & 1,1 & 840 & 0,2 \\
\hline 16 a 20 & 92.714 & 11,3 & 68.984 & 20,4 & 23.730 & 4,9 \\
\hline 21 a 25 & 158.112 & 19,2 & 100.840 & 29,8 & 57.272 & 11,5 \\
\hline 26 a 30 & 209.017 & 25,4 & 77.613 & 22,9 & 131.404 & 27,1 \\
\hline 31 a 35 & 219.390 & 26,7 & 50.581 & 14,9 & 168.809 & 34,8 \\
\hline 36 a 40 & 111.998 & 13,6 & 26.634 & 7,9 & 85.364 & 17,6 \\
\hline 410 más & 22.585 & 2,7 & 6.462 & 1,9 & 16.123 & 3,3 \\
\hline Sin información de edad materna & 4.363 & 0,5 & 3.410 & 1,0 & 953 & 0,2 \\
\hline \multicolumn{7}{|l|}{ Instrucción materna } \\
\hline Primario incompleto o menor & 18.097 & 2,2 & 14.387 & 4,3 & 3.710 & 0,8 \\
\hline Primario completo & 163.626 & 19,9 & 136.972 & 40,5 & 26.654 & 5,5 \\
\hline Secundario incompleto & 114.367 & 13,9 & 71.614 & 21,2 & 42.753 & 8,8 \\
\hline Secundario completo & 222.874 & 27,1 & 81.396 & 24,1 & 141.478 & 29,2 \\
\hline Universitario incompleto & 69.771 & 8,5 & 9.691 & 2,9 & 60.080 & 12,4 \\
\hline Universitario completo & 212.512 & 25,8 & 8.632 & 2,6 & 203.881 & 42,1 \\
\hline EGB polimodal incompleto/completo & 5.905 & 0,7 & 2.392 & 0,7 & 3.513 & 0,7 \\
\hline Sin información de instrucción materna & 15.739 & 1,9 & 13.313 & 3,9 & 2.426 & 0,5 \\
\hline \multicolumn{7}{|l|}{ Edad gestacional (en semanas) } \\
\hline$<$ a 34 & 17.597 & 2,1 & 6.793 & 2,0 & 10.804 & 2,2 \\
\hline $34-35-36$ & 49.511 & 6,0 & 16.959 & 5,0 & 32.552 & 6,7 \\
\hline 37 & 60.953 & 7,4 & 19.002 & 5,6 & 41.951 & 8,7 \\
\hline 38 & 159.347 & 19,4 & 47.666 & 14,1 & 111.681 & 23,1 \\
\hline 39 & 216.483 & 26,3 & 86.705 & 25,6 & 129.778 & 26,8 \\
\hline 40 & 243.008 & 29,5 & 123.518 & 36,5 & 119.490 & 24,7 \\
\hline 41 & 60.024 & 7,3 & 28.861 & 8,5 & 31.163 & 6,4 \\
\hline$>a 41$ & 4.527 & 0,6 & 2.749 & 0,8 & 1.778 & 0,4 \\
\hline Sin información de edad gestacional & 11.441 & 1,4 & 6.143 & 1,8 & 5.298 & 1,1 \\
\hline
\end{tabular}

Fuente: Elaboración propia a partir de la base de datos del Informe Estadístico de Nacido Vivo, Dirección de Estadística e Información en Salud, Ministerio de Salud de la Nación.

Nota: Se presentan 822.891 nacimientos institucionales, porque fueron excluidos del periodo 2004-2013 los nacimientos ocurridos en vivienda (domicilio particular), u otro lugar (vía publica, transporte u otro lugar) o nacimientos que no se pudo establecer el lugar de ocurrencia. $\mathrm{EGB}=$ Educación general básica.

$82,5 \%$ nace de lunes a viernes mientras que, en establecimientos públicos, es el $77,9 \%$. En la semana 38, esta diferencia se acentúa encontrando en los establecimientos privados que el $84,6 \%$ nacen de lunes a viernes, mientras que en los establecimientos públicos nace el $78,0 \%$.

Las edades gestacionales que superan el $20,0 \%$ de los nacimientos entre sábados y domingos en los establecimientos privados se corresponden a la semana 40 de edad gestacional, que son las de menos posibilidades de programar el inicio del parto.

En cambio, en los establecimientos públicos (aún sin alcanzar el patrón de distribución uniforme discreta) en todas las edades gestacionales, los nacimientos que ocurren sábados y domingos superan el 20,0\%. 
Tabla 2. Número de nacimientos institucionales de 34-41 semanas de edad gestacional y distribución porcentual de los días de la semana según establecimientos públicos o privados. Ciudad Autónoma de Buenos Aires, 2004 - 2013.

\begin{tabular}{|c|c|c|c|c|c|c|c|c|}
\hline \multirow{3}{*}{$\begin{array}{l}\text { Edad gestacional } \\
\text { (semanas) }\end{array}$} & \multicolumn{4}{|c|}{ Establecimientos públicos } & \multicolumn{4}{|c|}{ Establecimientos privados } \\
\hline & \multicolumn{2}{|c|}{ Lunes a viernes } & \multicolumn{2}{|c|}{ Sábado a domingo } & \multicolumn{2}{|c|}{ Lunes a viernes } & \multicolumn{2}{|c|}{ Sábado a domingo } \\
\hline & $n$ & $\%$ & $n$ & $\%$ & $n$ & $\%$ & $n$ & $\%$ \\
\hline 34,35 y 36 & 12.866 & 77,6 & 3.709 & 22,4 & 25.707 & 80,7 & 6.160 & 19,3 \\
\hline 37 & 14.493 & 77,9 & 4.105 & 22,1 & 33.854 & 82,5 & 7.168 & 17,5 \\
\hline 38 & 36.273 & 78,1 & 10.200 & 22,0 & 92.235 & 84,6 & 16.765 & 15,4 \\
\hline 39 & 62.976 & 74,5 & 21.506 & 25,5 & 103.167 & 81,5 & 23.464 & 18,5 \\
\hline 40 & 88.837 & 73,8 & 31.527 & 26,2 & 92.687 & 79,5 & 23.850 & 20,5 \\
\hline 41 & 20.789 & 74,1 & 7.282 & 25,9 & 24.964 & 82,2 & 5.407 & 17,8 \\
\hline Total & 236.234 & 75,1 & 78.329 & 24,9 & 372.614 & 81,8 & 82.814 & 18,2 \\
\hline
\end{tabular}

Fuente: Elaboración propia a partir de la base de datos del Informe Estadístico de Nacido Vivo, Dirección de Estadística e Información en Salud, Ministerio de Salud de la Nación.

Nota: Se presentan 769.991 nacimientos institucionales, porque se excluyeron los siguientes nacimientos: a) edad gestacional menor a 34 semanas o mayor a 41 semanas; b) cuando no se informaba la edad gestacional; c) cuando no figuraba en la fecha de nacimiento el día de la semana de ocurrencia.

En los siguientes gráficos, analizamos la distribución de los nacimientos por días de la semana en los establecimientos públicos o privados de acuerdo con las edades gestacionales de los recién nacidos. En la Figura 1, analizamos la distribución en números absolutos de los nacimientos en establecimientos públicos y privados entre las semanas seleccionadas para la investigación (semanas 34 a 41). Allí se observa que los nacimientos que ocurren en los establecimientos privados presentan una disminución los sábados y los domingos en forma más pronunciada que lo que se observa en los establecimientos públicos.

En la Figura 2 y la Figura 3, analizamos la distribución porcentual de los nacimientos en establecimientos públicos y privados. Para analizar la distribución porcentual por día se presentan los datos en porcentajes en el eje de las ordenadas, analizando el porcentaje correspondiente a cada día para cada tipo de establecimientos (del 100\% de los nacimientos en los establecimientos privados, se observa la distribución porcentual de cada día y

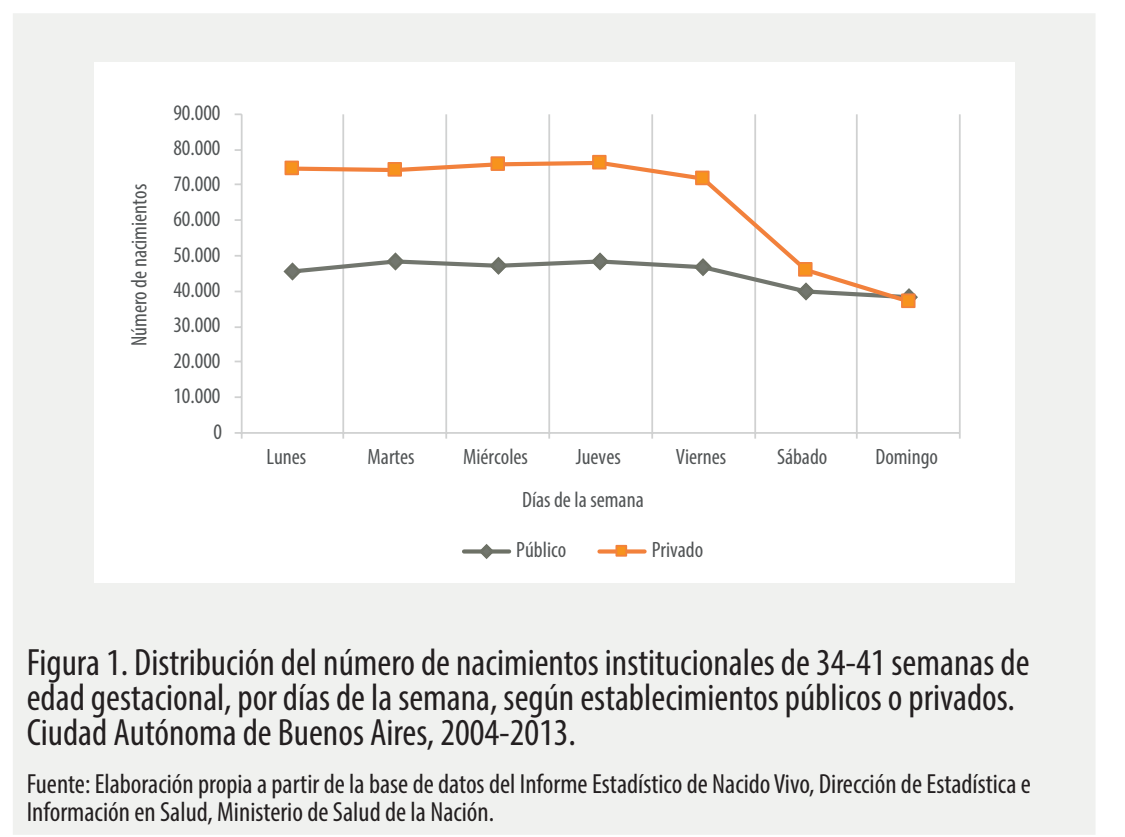




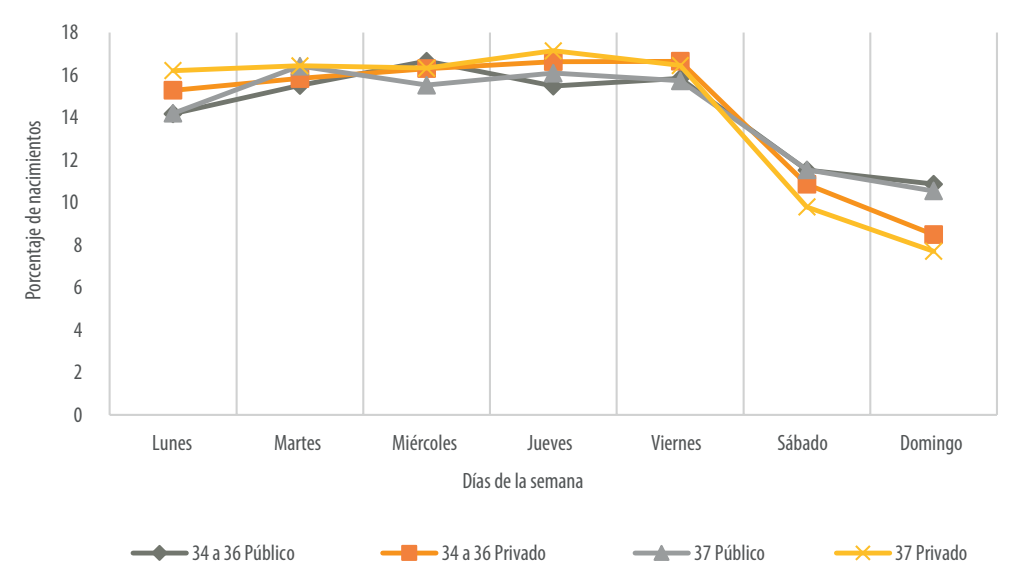

Figura 2. Distribución porcentual de los nacimientos institucionales ocurridos entre $34-36$ y 37 semanas de edad gestacional, por días de la semana, según establecimientos públicos o privados. Ciudad Autónoma de Buenos Aires, 2004-2013.

Fuente: Elaboración propia a partir de la base de datos del Informe Estadístico de Nacido Vivo, Dirección de Estadística e Información en Salud, Ministerio de Salud de la Nación.

del $100 \%$ de los nacimientos que ocurren en establecimientos públicos se presenta la distribución porcentual por día en ambas figuras). En la Figura 2, se muestra la distribución porcentual de los nacimientos pretérmino tardíos (semanas 34,35 y 36) y los nacimientos de término precoz (semana 37) en establecimientos públicos y privados. Así observamos que, para cada día, de lunes a viernes, el porcentaje de nacimientos varía entre el 14,2 y el
$17,1 \%$ para ambos establecimientos; en cambio, el porcentaje de nacimientos desciende los sábados y domingos, alcanzando en los nacimientos pretérminos tardíos en establecimientos privados un $10,8 \%$ los sábados y un $8,5 \%$ los domingos, mientras el porcentaje en los establecimientos públicos es del $11,5 \%$ los sábados y el 10,9\% los domingos.

En la Figura 3 observamos la distribución porcentual por días de la semana de los

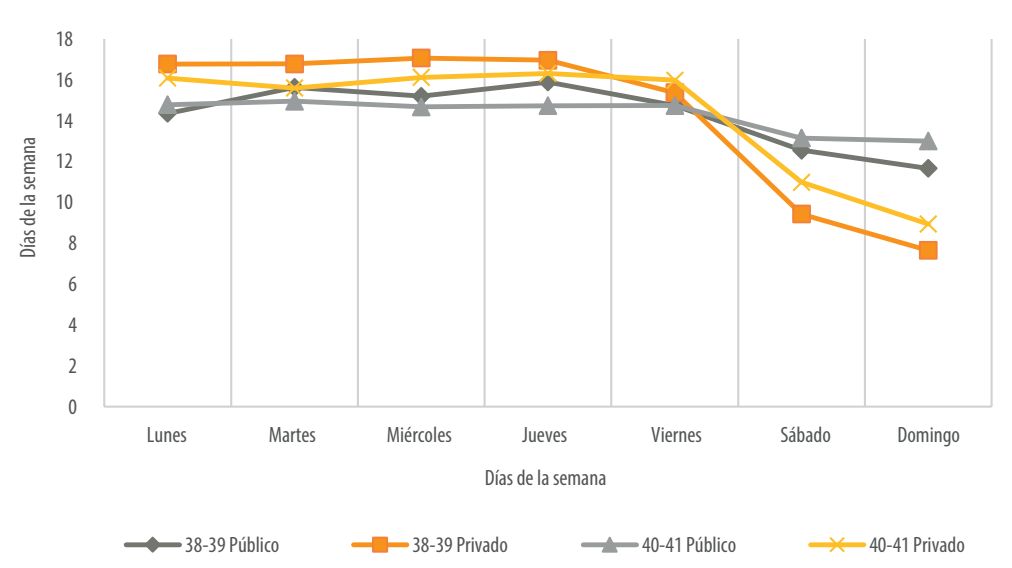

Figura 3. Distribución porcentual de los nacimientos institucionales ocurridos entre 38-39 y 40-41 semanas de edad gestacional, por días de la semana, según establecimientos públicos o privados. Ciudad Autónoma de Buenos Aires, 2004-2013.

Fuente: Elaboración propia a partir de la base de datos del Informe Estadístico de Nacido Vivo, Dirección de Estadística e Información en Salud, Ministerio de Salud de la Nación. 
nacimientos de término y observamos que, en los establecimientos privados, se produce un descenso de los nacimientos los días sábados y domingos. En los nacimientos ocurridos en establecimientos privados, en las semanas 38 y 39 , el porcentaje de nacimientos los días sábados alcanza el 9,4\% y los domingos el $7,7 \%$, contrastando con lo ocurrido de lunes a viernes, que en cada día el porcentaje supera el 15,4\%. En los establecimientos públicos, el descenso es menor, y alcanza el $12,5 \%$ los sábados y el 11,7\% los domingos. En los nacimientos de término completo (semanas 40 y 41), en los establecimientos públicos el porcentaje de distribución varía entre el 13,0\% y el 14,9\%; en cambio, en los establecimientos privados, los sábados y domingos hay una disminución: los sábados llega al 11,0\% de los nacimientos y los domingos al 8,9\%.

En el sector privado la proporción de nacimientos los días sábados y domingos, con relación a los demás días, es menor que en el sector público. Esta diferencia entre los dos sectores es estadísticamente significativa en todos los grupos de edad gestacional (test chi cuadrado con valor de $p<000$ ). En los establecimientos públicos y en los privados se encuentra una asociación positiva entre el grupo con mayor nivel de instrucción materna y los nacimientos ocurridos las días sábados y domingos (test chi cuadrado con valor de $p<000$ ).

\section{DISCUSIÓN}

En nuestra investigación se observa que la distribución de los nacimientos por días de la semana no es uniforme, hay una disminución los sábados y domingos (Figura 1). Estos resultados guardan relación con el estudio en Valencia (España), en el que los autores analizaron 3.675.110 nacimientos desde 1940 a 2010 y observaron en las primeras tres décadas una distribución semanal uniforme y a partir de la década de 1970 un incremento en los nacimientos de lunes a viernes y una disminución durante los sábados y domingos; tendencia que aumentó a partir del año $2000^{(1)}$.
Otras tres investigaciones concuerdan con el incremento de los nacimientos de lunes a viernes, pero difieren en las explicaciones. En Dinamarca, analizan 171.009 nacimientos ocurridos entre el año 2000 y 2009, excluyen los nacimientos resultantes de intervenciones obstétricas -como la cesárea electiva o los partos inducidos- y encuentran una variación semanal significativa, que la atribuyen al tiempo de admisión de las mujeres en la sala de parto y al número de personal disponible ${ }^{(17)}$. En Noruega, analizan 50.017 nacimientos desde 1990 a 2014, y encuentran que la frecuencia de nacimientos es más baja durante los fines de semana, y postulan como explicación que las mujeres embarazadas tienen diferentes maneras de vivir los fines de semana y los días de semana, con otro efecto en el inicio del parto. Además, como causas, señalan que las prácticas de atención perinatal pueden diferir ligeramente y que las mujeres son referidas con mayor frecuencia a otros hospitales los fines de semana ${ }^{(18)}$.

En el Reino Unido, se analizan 1.349.599 nacimientos entre los años 2010 y 2012, y encuentran que la distribución no es uniforme y que los nacimientos disminuyen los sábados y domingos, la explicación que adoptan es poner énfasis en el riesgo aumentado de nacer los fines de semana por las diferencias en la calidad de atención obstétrica(19).

En relación con el análisis de la distribución de los días de nacimientos en establecimientos públicos o privados (Figuras 1, 2 y 3), observamos que en ambos disminuyen los fines de semana, pero en los establecimientos privados, la diferencia es más marcada, lo cual nos permite postular que en los establecimientos privados hay mayor programación de los partos y nacimientos, incluyendo las cesáreas.

Según un informe del Ministerio de Salud de la Ciudad Autónoma de Buenos Aires en los hospitales públicos de la ciudad entre los años 2005 y 2013 los nacimientos por cesáreas alcanzaron el $26,7 \%{ }^{(20)}$, lo cual duplica lo recomendado por la OMS. En nuestro análisis, los nacimientos en establecimientos públicos de CABA, alcanzan el $41,1 \%$ del total (Tabla 1 ), si tenemos en cuenta la mayor concentración de los nacimientos de lunes a viernes en los 
establecimientos privados nos permite inferir que la tasa de cesáreas en ellos es aún mayor.

América del Sur tiene la tasa de cesáreas más alta del mundo con el $42 \%{ }^{(21)}$, con predominio de las cesáreas electivas ${ }^{(22)}$. La OMS describe que la tasa de cesáreas por encima de un $10 \%$ a $15 \%$ no se correlaciona con una mayor disminución de las tasas de mortalidad materna y neonatal ${ }^{(11)}$. En un estudio realizado en 24 países para analizar la idoneidad de las cesáreas realizadas sin indicaciones médicas se concluyó que su uso excesivo acarrea resultados maternos graves ${ }^{(22)}$. Un estudio ecológico que analiza 2.178.714 cesáreas, entre los años 1995 a 1997 en 12 países de Latinoamérica, concluye que se realizaron 850.000 "cesáreas innecesarias" en América Latina ${ }^{(23)}$.

Respecto de los nacimientos de término temprano o "nacidos demasiado pronto" (semana 37), en la Figura 2 se observa que son más frecuentes en los establecimientos privados y presentan la mayor diferencia en la distribución semanal. En un estudio multicéntrico que evalúa 24.077 nacimientos entre 1999 al 2002, en 19 centros de EEUU, se analizan las muertes neonatales o cualquier resultado adverso, y encuentran que los riesgos fueron mayores para el parto a las 37 semanas de gestación que para el de las 38 semanas, relación que se mantiene al comparar la semana 38 con la $39^{(24)}$.

En una revisión realizada en América Latina, encuentran mayor morbilidad respiratoria en los recién nacidos a término precoz respecto de los de término completo, y un mayor riesgo de complicaciones respiratorias neonatales que puede requerir cuidados intensivos, oxigenoterapia o asistencia respiratoria ${ }^{(25)}$.

Los riesgos de los nacimientos precoces son señalados en un estudio de cohorte, en el que analizaron 35.539 recién nacidos entre 1992 y 2011 en Barcelona y concluyen que la población de recién nacidos a término precoz (early term) presenta una mayor morbilidad neonatal respecto de la población de recién nacidos a término completo (full term), incluso tras haber excluido enfermedad materna ${ }^{(26)}$.

Los nacimientos tempranos presentan aristas de medicalización que pueden ser iatrogénicas para los recién nacidos. Nos en- contramos ante un abordaje de los partos que ha centrado sus expectativas en la disminución de riesgos, realizando una serie de intervenciones biotecnológicas protocolizadas que cuando su uso es innecesario pueden incrementar los riesgos ${ }^{(10)}$.

Entre las potenciales limitaciones de esta investigación, se pueden identificar los siguientes aspectos: a) los días feriados o no laborales no fueron considerados en el análisis porque su dimensión era menor en el periodo considerado; b) la estacionalidad no fue analizada porque en un análisis exploratorio inicial no se encontraron diferencias relevantes y porque no era el objeto principal del artículo; c) la edad gestacional, informada en la base de datos del Informe Estadístico de Nacido Vivo, debería ser colocada a partir de la fecha de la última menstruación, pero cuando no se cuenta con esa información se coloca la edad gestacional por el examen clínico (método de Capurro). Ninguna de las limitaciones señaladas modificaría sustancialmente los resultados presentados.

Esta investigación describe aristas de la medicalización del proceso de parto y nacimiento. Al no contar en las fuentes de información con la vía de finalización del parto, ni con el dato de inducción, la programación del parto se mide en forma indirecta según los días de la semana en que ocurren. Lo anterior, aporta un aspecto poco explorado, al describir los nacimientos por día de la semana como una forma de cuantificar un aspecto que caracteriza la expansión de la medicalización del parto, una de las transformaciones centrales ocurridas en la última mitad del siglo pasado en este fenómeno natural ${ }^{(27)}$.

La discusión anterior nos lleva a interrogarnos si los nacimientos responden más a las necesidades y a las agendas de las y los profesionales que a la evolución natural de parir y la salud de las niñas y los niños recién nacidos. Por todo ello concluimos que el momento del nacimiento se ha convertido en un acontecimiento socialmente controlado ${ }^{(1)}$, en el que las mujeres devienen objetos del control que ejercen las instituciones de la medicina moderna sobre ellas y sus cuerpos. 


\section{REFERENCIAS BIBLIOGRAFICAS}

1. Lledó J, Pavía JM, Morillas Jurado FG. Transformaciones en la distribución semanal de nacimientos: Un análisis temporal 1940-2010. Revista Española de Investigaciones Sociológicas. 2017;(159):151-162. doi: 10.5477/cis/reis.159.151.

2. Castrillo B. De partos y derechos en el camino hacia la humanización. VIII Jornadas de Investigación en Antropología Social Santiago Wallace, 27 al 29 de julio de 2016, Ciudad Autónoma de Buenos Aires, Argentina.

3. Ronda E, Hernández-Mora A, García AM, Regidor E. Ocupación materna, duración de la gestación y bajo peso al nacimiento. Gaceta Sanitaria. 2009;23(3):179-185.

4. Maroto-Navarro G, García-Calvente M, Mateo-Rodríguez I. El reto de la maternidad en España: dificultades sociales y sanitarias. Gaceta Sanitaria. 2004;18(5):13-23.

5. Menéndez EL. El modelo médico y la salud de los trabajadores. Salud Colectiva. 2005;1(1):9-32. doi: 10.18294/ sc.2005.1.

6. García Jordá, Díaz Bernal Z, Acosta Álamo M. El nacimiento en Cuba: análisis de la experiencia del parto medicalizado desde una perspectiva antropológica. Ciência \& Saúde Coletiva. 2012;17(7):1893-1902.

7. Hernández Garre JM, Echevarría Pérez P. El nacimiento hospitalario e intervencionista: un rito de paso hacia la maternidad. AIBR, Revista de Antropología Iberoamericana. 2015;10(3):401-426.

8. Montes Muñoz MJ. Las culturas del nacimiento representaciones y prácticas de las mujeres gestantes, comadronas y médicos. [Tesis de doctorado]. Tarragona: Universitat Rovira i Virgili; 2007.

9. Azeredo YN, Schraiber LB. El poder médico y la crisis de los vínculos de confianza en la medicina contemporánea. Salud Colectiva. 2016;12(1):9-21. doi: 10.18294/ sc. 2016.864

10. Hernández Garre JM, Echevarría Pérez P, Gomariz Sandoval MJ. ¿Némesis obstétrica o disminución del riesgo? A debate bioético el abordaje intervencionista en los partos de bajo riesgo. Acta Bioethica. 2017;23(1):161-170.

11. Organización Mundial de la Salud. Recomendaciones de la OMS para la conducción del trabajo de parto. Ginebra: OMS; 2015.

12. Mathai $M$, Hofmeyr GJ. Abdominal surgical incisions for caesarean section. Cochrane Database of Systematic Reviews. 2003;(4):CD004453. doi: 10.1002/14651858. CD004453.

13. Ministerio de Salud de la Nación, Dirección de Estadísticas e Información en Salud. Formularios estadísticos: 2. Nacido vivos [Internet]. 2016 [citado 6 dec 2019]. Disponible en: https://tinyurl.com/y4rnf5jb
14. Organización Mundial de la Salud. Nacidos demasiado pronto: Informe de Acción Global sobre Nacimientos Prematuros [Internet]. 2012 [citado 6 dic 2019]. Disponible en: https://tinyurl.com/y3ap4ae6.

15. Tita ATN, Landon MB, Spong CY, Lai $Y$, Leveno $\mathrm{KJ}$, Varner $\mathrm{MW}$, et al. Timing of elective repeat cesarean delivery at term and neonatal outcomes. New England Journal of Medicine. 2009;360(2):111-120. doi: 10.1056/NEJMoa0803267.

16. Argentina. Ley 17622, Será modernizado y racionalizado el servicio estadístico - Créase el Instituto Nacional de Estadística y Censos [Internet]. 1968 [citado 19 nov 2019]. Disponible en: https://tinyurl.com/y3qgw8h2.

17. Gam CMB, Tanniou J, Keiding N, Løkkegaard EL. A model for the distribution of daily number of births in obstetric clinics based on a descriptive retrospective study. BMJ Open. 2013;3(8):e002920. doi: 10.1136/ bmjopen-2013-002920.

18. Barra M, Lindstrøm JC, Adams SS, Augestad LA. Sesongjusterte fødselsfrekvenser er Poisson-fordelte. Tidsskriftet. 2015;135(23/24):2154-2158.

19. Palmer WL, Bottle A, Aylin P. Association between day of delivery and obstetric outcomes: observational study. BMJ. 2015;351:h5774. doi: 10.1136/bmj.h5774.

20. Ministerio de Salud (GCBA), Subgerencia Operativa Estadísticas de Salud. Partos realizados en hospitales del Gobierno de la Ciudad de Buenos Aires por tipo de parto según hospital, Ciudad de Buenos Aires, Años 2005/2015 [Internet]. 2016 [citado 25 jun 2019]. Disponible en: https://tinyurl.com/y46tdf6v.

21. Betrán AP, Ye J, Moller A-B, Zhang J, Gülmezoglu $A M$, Torloni MR. The increasing trend in caesarean section rates: Global, regional and national estimates: 1990-2014. PLoS One. 2016;11(2):e0148343. doi: 10.1371/journal.pone.0148343.

22. Souza J, Lumbiganon $P$, Laopaiboon $M$, Carroli $G$, Fawole B, Ruyan P, et al. Caesarean section without medical indications is associated with an increased risk of adverse short-term maternal outcomes: the 2004-2008 WHO Global Survey on Maternal and Perinatal Health. BMC Medicine. 2010;8:71. doi: 10.1186/1741-7015-8-71.

23. Belizan JM, Althabe F, Barros FC, Alexander $S$ Showalter E, Griffin A, et al. Rates and implications of caesarean sections in Latin America: ecological study Commentary: all women should have a choice Commentary: increase in caesarean sections may reflect medical control not women's choice Commentary: «health has become secondary to a sexually attractive body". BMJ. 27 de noviembre de 1999;319(7222):1397. doi: 10.1136/bmj.319.7222.1397.

24. Tita ATN, Landon MB, Spong CY, Lai Y, Leveno $\mathrm{KJ}$, Varner $\mathrm{MW}$, et al. Timing of elective repeat cesarean delivery at term and neonatal outcomes. New England Journal of Medicine. 2009;360(2):111-120. doi: 10.1056/NEJMoa0803267. 
25. Mariani GL, Vain NE. The rising incidence and impact of non-medically indicated pre-labour cesarean section in Latin America. Seminars in Fetal and Neonatal Medicine. 2019;24(1):11-17. doi: 10.1016/j. siny.2018.09.002.

26. Martínez-Nadal S, Demestre X, Raspall F, Álvarez JA, Elizari MJ, Vila C, et al. Morbilidad neonatal en los recién nacidos a término precoz. Anales de Pediatría. 2014;81(1):39-44. doi: 10.1016/j.anpedi.2013.10.015.

27. Conrad P. Medicalization and social control. Annual Review of Sociology. 1992;18:209-232. doi: 10.1146/ annurev.so.18.080192.001233.

\section{FORMA DE CITAR}

Rosemberg P, Alazraqui M, Spinelli H. "Las atendemos de lunes a viernes": nacimientos según días de la semana en la Ciudad Autónoma de Buenos Aires, 2004-2013. Salud Colectiva. 2020;16:e3079. doi: 10.18294/sc.2020.3079.

Recibido: 7 ago 2020 | Versión final: 6 oct 2020 | Aprobado: 20 oct 2020 | Publicado en línea: 11 nov 2020

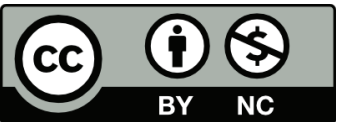

Esta obra está bajo una licencia de Creative Commons Reconocimiento-NoComercial 4.0 Internacional. Reconocimiento - Permite copiar, distribuir y comunicar públicamente la obra. A cambio, se debe reconocer y citar al autor original. No Comercial - Esta obra no puede ser utilizada con finalidades comerciales, a menos que se obtenga el permiso. 